\title{
De compras para salud: Granos integrales ${ }^{1}$
}

\author{
Wendy J. Dahl y Lauren Foster ${ }^{2}$
}

\section{Granos integrales vs. Granos refinados}

Los granos incluyen alimentos como el pan, la pasta, la avena, y las galletas. Cualquier alimento hecho con trigo, avena, arroz, maíz, cebada u otro cereal es considerado un grano (1).

Los granos integrales contienen el grano entero. Se usa poco procesamiento en la producción de alimentos integrales.

Los granos refinados han sido procesados para remover el germen y el salvado (vea el diagrama abajo). Este proceso también remueve mucha de la fibra que contiene el grano. Las vitaminas del complejo $\mathrm{B}$, que han sido removidas en este proceso, necesitan ser añadidas de nuevo a través del enriquecimiento.

\section{¿Cuantos granos necesitamos?}

Las porciones recomendadas de granos dependen de la edad, el genero y cuanta energía se necesite. El departamento de agricultura de los Estados Unidos (USDA) recomienda que la mitad de la porción diaria de granos sea de granos integrales para todas las personas de todas las edades, con un equivalente a $3 \mathrm{oz}$ de granos integrales

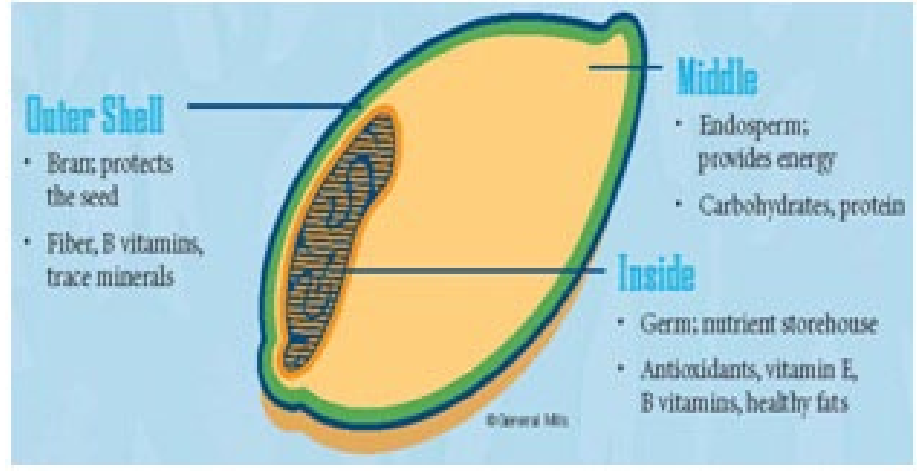

Figura 1. Los granos enteros tienen todavía las tres partes naturales del grano de grano intacto el exterior salvado rico en fibra o capa exterior, el endospermo amiláceo que protege, y el germen lleno de nutrientes, que proporciona alimento a la semilla. Reproducido con el permiso de General Mills.

cada día. El equivalente de una onza es comparable a una tajada de pan, una taza de cereal o media taza de arroz o pasta cocida. En la tabla 1 está el consumo diario de granos recomendado para individuos con actividad física moderada (1).

\section{Comprando granos integrales}

Una de las mejores formas para identificar los alimentos que contienen granos integrales es buscar la "estampa de granos integrales." Una "estampa de granos integrales" en un paquete de comida identifica a los alimentos aprobados por el cónsul de granos integrales. El Whole Grains Council

1. The English version of this document is FSHN10-13/FS161 Shopping for Health: Whole Grains. Este documento, FSHN10-13s, es uno de una serie de publicaciones del Departamento de Food Science and Human Nutrition, Servicio de Extensión Cooperativa de la Florida, Instituto de Alimentos y Ciencias Agrícolas, Universidad de la Florida. (UF/IFAS). Fecha de primera publicación enero de 2013. Visite el sitio web de EDIS en http://edis.ifas.ufl. edu/.

2. Wendy J. Dahl, PhD, assistant professor, y Lauren Foster, BS; Food Science and Human Nutrition Department, University of Florida; Gainesville, FL 32611.

El uso de nombres comerciales citados en esta publicación es sólo con el propósito de brindar información específica. El Instituto (UF/IFAS) no garantiza los productos nombrados, y las referencias a ellos en esta publicación no significan nuestra aprobación a la exclusión de otros productos de composición comparable. 
recomienda que usted aspire a comer 48 gramos de granos integrales cada día (2).

Hay 2 tipos de sellos:

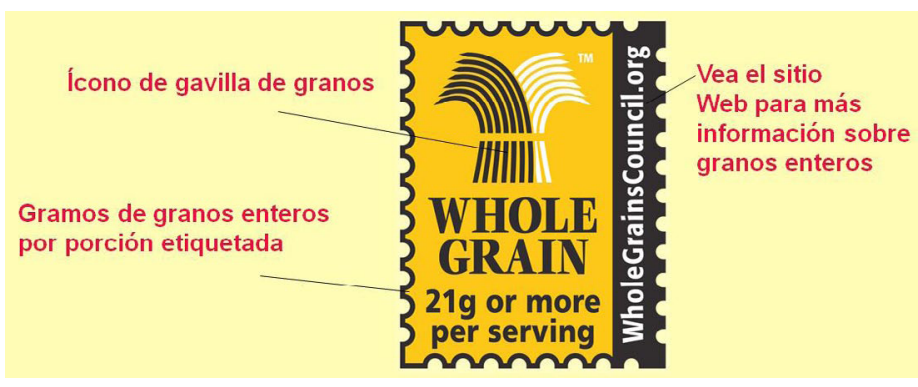

Figura 2. El sello "básico"

La marca del $100 \%$ aparece si todos los granos* que contiene el producto son granos enteros; Es decir, que NO contiene granos refinados

\section{El sello del "100\%"}

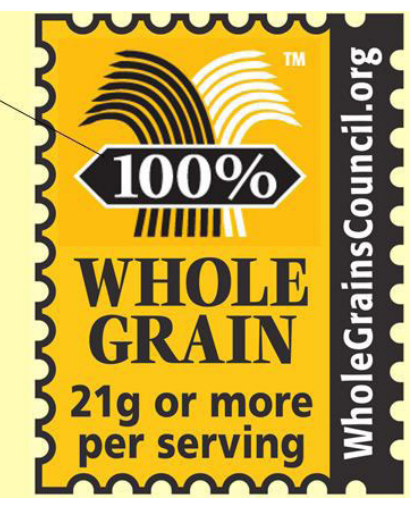

Figura 3. El sello del "100\%"

La lista de ingredientes en las etiquetas de los productos también ayuda a identificar los granos integrales. Si los granos integrales están listados como el primer ingrediente del producto, es probable que sea una buena fuente de granos integrales. Estos son algunos ejemplos de granos integrales: trigo integral, avena o harina integral, maíz de grano entero, palomitas de maíz, arroz integral, centeno integral y la cebada de grano entero.

Tabla 1. Consumo de granos diario recomendado

\begin{tabular}{|c|c|c|}
\hline Etapa de la vida & $\begin{array}{c}\text { Recomendación } \\
\text { de granos } \\
\text { (equivalente en } \\
\text { onzas al día) }\end{array}$ & $\begin{array}{c}\text { Recomendación de } \\
\text { los granos integrales } \\
\text { (equivalente en } \\
\text { onzas al día) }\end{array}$ \\
\hline Niños, 2-3 & 3 & 1.5 \\
\hline Niños, 4-8 & $4-5$ & $2-2.5$ \\
\hline Mujeres, 9-18 & $5-6$ & 3 \\
\hline Hombres, 9-18 & $6-7$ & $3-3.5$ \\
\hline Mujeres, 19+ & $5-6$ & 3 \\
\hline Hombres, 19+ & $6-8$ & $3-4$ \\
\hline
\end{tabular}

Abajo esta listado un ejemplo de los ingredientes de un producto de granos enteros que fue tomada del cereal de Kelloggs ${ }^{\circledast}$ Frosted Mini-Wheats ${ }^{\circledast}$.

INGREDIENTES: Trigo integral, azúcar, jarabe de maíz de alta fructosa, gelatina, hierro reducido, niacina mida, óxido de zinc...

\section{De compras para la merienda}

Muchas personas meriendan con alimentos procesados en vez de alimentos de granos integrales. ¡Los granos integrales tienen mas fibra que los granos refinados lo cual puede satisfacer más su apetito y mantenerlo lleno por más tiempo!

Tabla 2. Alimentos de merienda con granos integrales

\begin{tabular}{|c|c|}
\hline Merienda & $\begin{array}{c}\text { Granos integrales por } \\
\text { porción }(\mathbf{g})\end{array}$ \\
\hline Frito-Lay ${ }^{\circledR}$ Sun Chips & 18 \\
\hline Galletas Kashi ${ }^{\circledast}$ TLC & 8 \\
\hline $\begin{array}{l}\text { Pretzels miniatura hechos con grano } \\
\text { integral Rold Gold }{ }^{\circledR}\end{array}$ & 8 \\
\hline Barras de Granola Cascadian Farm ${ }^{\oplus}$ & 8 \\
\hline Barras de Cereal Nutri-Grain ${ }^{\circledR}$ & 8 \\
\hline Chips de tortilla Tostito ${ }^{\oplus}$ & 8 \\
\hline General Mills ${ }^{\circledast}$ Cheerios Snack Mix & 8 \\
\hline \multicolumn{2}{|l|}{$\mathbf{g}=$ gramos } \\
\hline
\end{tabular}

Muchas marcas de galletas y otras meriendas ofrecen una variedad de productos hechos con granos integrales. Abajo están listadas algunas meriendas que traen "la estampa de granos integrales." Cuando esté comprando las meriendas, lea las etiquetas de los alimentos cuidadosamente porque las marcas frecuentemente cargan variedades que pueden no tener la misma cantidad de granos integrales.

\section{De compras para la cena}

Ir de compras por alimentos listos para cocinar que contengan granos integrales puede parecer difícil, pero existen varias pastas integrales disponibles en el mercado y también platos fuertes y acompañantes que incorporan granos integrales. En la tabla 3 están listados algunos ejemplos de platos fuertes y acompañantes que contienen granos integrales. 


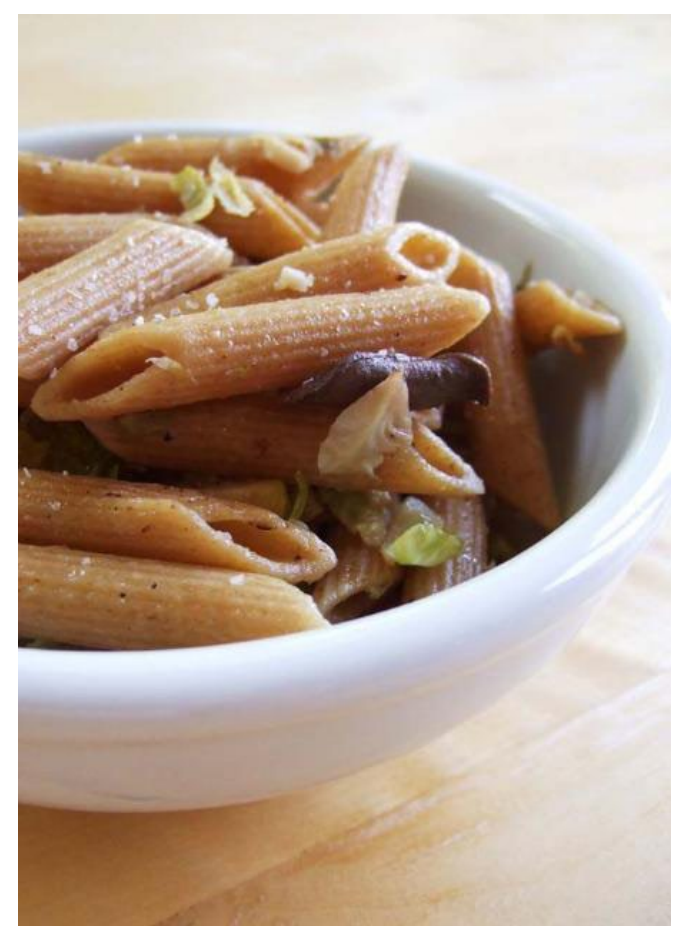

Figura 4. Imagen de pasta integral con las coles de Bruselas y las setas. $\sim$ Foto por Bryan Ochalla bajo una licencia de Creative Commons Reconocimiento-Compartir bajo la misma licencia 2.0.

Tabla 3. Comidas principales de granos integrales

\begin{tabular}{|c|c|}
\hline Platos fuertes & $\begin{array}{c}\text { Granos integrales por } \\
\text { porción }(\mathbf{g})\end{array}$ \\
\hline $\begin{array}{l}\text { Linguini de } 100 \% \text { grano integral } \\
\text { Buitoni }^{\circledR}\end{array}$ & 62 \\
\hline $\begin{array}{l}\text { Raviolis de cuatro quesos de } 100 \% \\
\text { grano integral Buitoni }{ }^{\circledR}\end{array}$ & 45 \\
\hline Pasta garden con vegetales Kashi ${ }^{\circledast}$ & 40 \\
\hline Pasta integral Barilla ${ }^{\circledR}$ & 28 \\
\hline $\begin{array}{l}\text { Mantequilla de maní y jalea de uva } \\
\text { Smucker's }{ }^{\circledR} \text { en grano integral }\end{array}$ & 16 \\
\hline Pizza congelada Kashi ${ }^{\circledast}$ & 8 \\
\hline $\begin{array}{l}\text { Pollo empanizado totalmente } \\
\text { cocinado Tyson }^{\circledast}\end{array}$ & 8 \\
\hline $\begin{array}{l}\text { Pasta con queso ricotta y espinaca de } \\
\text { Weight Watchers }{ }^{\circledR}\end{array}$ & 8 \\
\hline \multicolumn{2}{|l|}{$\mathbf{g}=$ gramos } \\
\hline
\end{tabular}

Tabla 4. Cereales para desayunos con granos integrales

\begin{tabular}{|c|c|}
\hline Cereal & $\begin{array}{l}\text { Granos integrales } \\
\text { por porción }(\mathbf{g})\end{array}$ \\
\hline Kellogg's ${ }^{\oplus}$ Mini-Wheats & 58 \\
\hline Kashi ${ }^{\circledast}$ Summer Berry Granola & 41 \\
\hline Heartland ${ }^{\circledast}$ Granola & 30 \\
\hline Kashi $^{\circledast}$ Go Lean Crunch & 17 \\
\hline Granola con fruta y maní Cascadian Farm ${ }^{\circledast}$ & 16 \\
\hline $\begin{array}{l}\text { General Mills }{ }^{\circledR} \text { Cheerios, Cheerios } \\
\text { integrales, Total, Wheaties }\end{array}$ & 16 \\
\hline $\begin{array}{l}\text { General Mills }{ }^{\circledast} \text { Cinnamon Toast Crunch, } \\
\text { Golden Grahams, Cocoa Puffs }\end{array}$ & 8 \\
\hline \multicolumn{2}{|l|}{$\mathbf{g}=$ gramos } \\
\hline
\end{tabular}

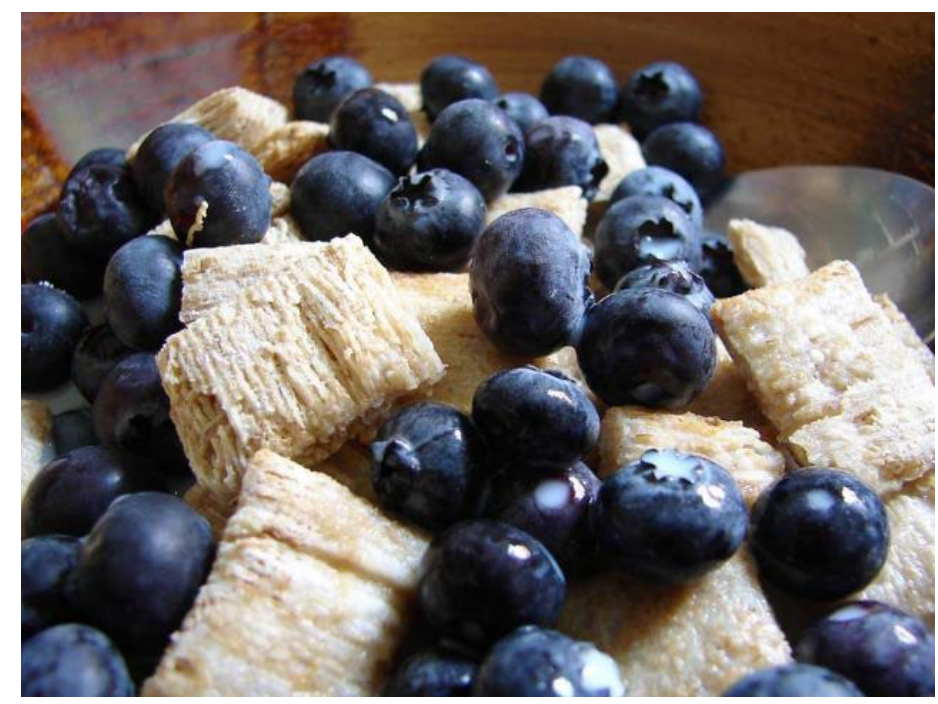

Figura 5. Imagen de cereal de grano entero cubierto con arándanos. Foto por Kendalia bajo una licencia Creative Commons Reconocimiento-No comercial License 2.0.

\section{De compras para el cereal de desayuno}

Consumir los suficientes granos integrales es cada vez más fácil ya que muchos cereales para desayunos ahora son hechos con granos integrales. En la tabla 4 están listados los ejemplos de cereales para desayunos que tienen "la estampa de granos integrales." Muchos más cereales para desayunos están hechos con granos integrales así que revise las etiquetas de sus cereales favoritos. 


\section{¡Sea un comprador informado!}

Sustituir los productos refinados por los de granos integrales puede ayudar con el manejo del peso y a disminuir el riesgo de enfermedades crónicas (3). ¡Cuando vaya de compras, lea las etiquetas de los alimentos y busque los que tengan "la estampa de granos integrales"!

Aquí hay algunos consejos para ayudarlo a incorporar los granos integrales en su día.

- Meriende con triángulos de pita integral y humus

- Consuma arroz integral en vez de arroz blanco

- Sustituya harina de trigo blanca por harina integral para hornear

- Escoja cereales integrales para el desayuno o la merienda de la tarde

\section{Referencias}

1. U.S. Department of Agriculture. MyPyramid. 1 Oct. 2009. Web. Accessed: 26 Feb. 2010. http://www.mypyramid. gov/pyramid/grains.html

2. Whole Grains Council. Whole Grain Stamp. Accessed: 28 March 2010. http://wholegrainscouncil.org/ whole-grain-stamp

3. U.S. Department of Health and Human Services and U.S. Department of Agriculture. Dietary Guidelines for Americans, 2005. 6th Edition, Washington, DC: U.S. Government Printing Office, January 2005. 